\title{
绚丽多彩的纳米复合材料
}

徐林 1 , 侯文华 2

1 南京师范大学化学与材料科学学院, 江苏省新型动力电池重点实验室, 南京 210023

2 南京大学化学化工学院, 介观化学教育部重点实验室, 南京 210023

\section{Diverse Fantastic Nanocomposites}

\author{
Lin $\mathrm{Xu}^{1}$, Wenhua Hou ${ }^{2}$ \\ ${ }^{1}$ Jiangsu Key Laboratory of New Power Batteries, School of Chemistry and Materials Science, Nanjing Normal University, \\ Nanjing 210023, P. R. China. \\ ${ }^{2}$ Key Laboratory of Mesoscopic Chemistry of MOE, School of Chemistry and Chemical Engineering, Nanjing University, \\ Nanjing 210023, P. R. China. \\ Emails: xulin001@njnu.edu.cn (L.X.); whou@nju.edu.cn (W.H.). \\ Published online: January 7, 2020.
}

在纳米尺度上复合物理化学性能差别迥异的 成分, 实现材料的多功能化是纳米合成的一个热 点领域。纳米复合材料体系中各组分之间可以产 生强相互耦合作用, 不仅可以增强材料各自的本 征特性, 而且还可能表现出许多新奇的物化特性, 从而突破单一组分材料性能的局限, 在新型功能 材料研发、能源转化和储存、环境保护与污染处理 和生物医药等领域具有广泛应用。随着合成技术 和表征手段的快速发展, 人们已可以在原子和分 子水平上控制纳米复合材料的合成, 观察复合材 料不同成分的界面结构, 揭示复合材料不同组分 间的耦合原理, 并调控复合材料的物化性能。本专 辑中收集了我国部分科学家在相关领域的研究成 果, 将展示一系列纳米复合材料的可控合成、结构 表征及其在光催化、电催化、能源储存等领域应用 方面的最新进展。

将不同化学组分在纳米尺度上进行可控整合 具有一定的挑战性。杨军等 ${ }^{1}$ 利用贵金属在 $\mathrm{Ag}_{2} \mathrm{~S}$ 纳 米颗粒中空位互换的扩散机制或半导体纳米颗粒 的自纯化机制, 将预合成的贵金属 $(\mathrm{Au} 、 \mathrm{Ag} 、 \mathrm{Pd}$ 和 $\mathrm{Pt}$ )和 $\mathrm{Ag}_{2} \mathrm{~S}$ 形成的核壳结构自发演变成由贵金属和 $\mathrm{Ag}_{2} \mathrm{~S}$ 构成的异质纳米二聚体结构, 如 $\mathrm{Au}-\mathrm{Ag}_{2} \mathrm{~S} 、 \mathrm{Ag}-$ $\mathrm{Ag}_{2} \mathrm{~S} 、 \mathrm{Pd}-\mathrm{Ag}_{2} \mathrm{~S}$ 和 $\mathrm{Pt}-\mathrm{Ag}_{2} \mathrm{~S}$ 。利用贵金属在半导体中 的迁移可以用来制备一些常规方法不易获得的异 质结构纳米材料。殷丽莎和谢小吉等 ${ }^{2}$ 采用共沉淀
法在 $\mathrm{NaGdF}_{4}: \mathrm{Yb} / \mathrm{Tm}$ 上转换纳米粒子表面包覆了 无定形的 $\mathrm{Y}(\mathrm{OH}) \mathrm{CO}_{3}$ 壳层, 并在一定温度下将 $\mathrm{Y}(\mathrm{OH}) \mathrm{CO}_{3}$ 壳层转化为结晶相的 $\mathrm{YOF}$ 。该方法可以 拓展于其他无定形壳层的包覆及蛋黄-蛋壳结构 纳米粒子的制备。

纳米复合材料中不同组分间的协同效应以及 界面效应等使其在电催化、能源存储和光催化等 领域中具有巨大的应用前景。张立学等 ${ }^{3}$ 以具有良 好导电性、电化学稳定以及耐腐蚀等优点的一维 多孔氮化钛 $(\mathrm{TiN})$ 纳米管为载体, 使用原子层沉积 技术(ALD)在TiN载体上沉积尺寸均一且高度分散 的Pt纳米粒子。得益于 $\mathrm{Pt}$ 纳米颗粒的高分散性、TiN 载体的高比表面积和导电性以及 $\mathrm{Pt}$ 与 $\mathrm{TiN}$ 之间的 相互作用, 所得复合催化剂在电催化氧气还原反 应(ORR)中表现出比商用 $\mathrm{Pt} / \mathrm{C}$ 催化剂更高的催化 活性和稳定性。冯立纲等 ${ }^{4}$ 以 $\mathrm{Pt}$ 纳米颗粒结合的 ZIF-67为前驱体, 在氮气保护下将其热解成氮掺 杂碳负载PtCo 合金纳米颗粒组成的PtCo-NC复合 催化剂。由于 $\mathrm{PtCo}$ 纳米颗粒与氮掺杂载体间的协 同效应, 所得 $\mathrm{PtCo}-\mathrm{NC}$ 复合催化剂在电催化料甲醇 和乙醇氧化反应中均表现出优异的活性、稳定性 和抗中毒能力。

吴平和周益明等 5 通过氧化石墨烯 $(\mathrm{GO})$ 和壳 聚糖 $(\mathrm{Cs})$ 之间的氢键以及静电作用形成 $\mathrm{GO}$ 水凝 胶, 从而将纳米硅颗粒和碳纳米管(CNT)原位包封 
于其中, 再经冷冻干燥及随后的热处理制得三维 硅/碳纳米管/石墨烯(Si-CNT@G)纳米复合材料。 当作为锂离子电池的负极材料时, 两种碳介质的 协同能有效缓冲硅材料在充放电过程中脱/嵌锂引 起的体积变化, 缩短了锂离子和电子传输的距离, Si-CNT@G复合材料表现出较好的循环稳定性以 及倍率性能。武祥等 6 采用简单的水热方法在泡沫 镍基底上生长了钴酸镍纳米片。合成的 $\mathrm{NiCo}_{2} \mathrm{O}_{4}$ 纳 米片直接用作超级电容器电极, 呈现出优异的电 化学性能和稳定性, 在未来储能器件中具有良好 的电化学应用前景。曹少文和余家国等7采用简单 的水热法并结合后续制烧处理的方法制备了锰钴 氧化物与碳纤维的复合材料。该方法制备的复合 材料中的锰钴氧化物可以均匀地分散在碳纤维表 面, 从而增加了电极材料与电解质的界面接触, 提 高了电极材料的利用率。因此, 碳纤维负载锰钴氧 化物的复合材料可用于提高超级电容器的电化学 性能, 并为制备高性能超级电容器的电极材料提 供了新的策略。

袁玉鹏等 ${ }^{8}$ 在氨氛围中热处理石墨相氮化碳 $\left(\mathrm{g}-\mathrm{C}_{3} \mathrm{~N}_{4}\right)$, 抑制氮原子的氧化程度、控制 $\mathrm{N}$ 空位浓 度和提高产物结晶度, 最终提升其光催化制氢的 性能。殷丽莎等 ${ }^{9}$ 以原位还原的方法, 在 $\mathrm{g}-\mathrm{C}_{3} \mathrm{~N}_{4}$ 纳 米片表面沉积Pt-M ( $\mathrm{M}=\mathrm{Co}, \mathrm{Ni}, \mathrm{Fe})$ 助催化剂, 以 降低Pt的用量, 提升光解水制氢的性能。刘超和戴 勇等 10 采用稳态共沉淀法制备了 $\mathrm{ZnCuAl}$ $\mathrm{LDH} / \mathrm{Bi}_{2} \mathrm{MoO}_{6}$ 纳米复合材料, 研究了所得材料在 可见光下降解污染物罗丹明 $B(R h B)$ 的光催化性 能, 并提出了一种可能的光催化机制。

纳米复合材料种类繁多, 包罗万象, 在诸多领 域都显示出巨大的发展空间。但有关纳米复合材 料的可控制备、表征手段、结构组分与性能之间的 构效关系等仍需深入探究。希望广大读者能从本 专刊的论文中有所启发和获益。

\section{References}

(1) Liu, D.; Chen, D.; Liu, H.; Yang, J. Acta Phys. -Chim. Sin. 2020, 36
(7), 1906069. [刘丹叶, 陈东, 刘卉, 杨军. 物理化学学报, 2020, 36 (7), 1906069.] doi: 10.3866/PKU.WHXB201906069

(2) Liu, D.; Chen, X.; Yuan, Z.; Lü, M.; Yin, L.; Xie, X.; Huang, L. Acta Phys. -Chim. Sin. 2020, 36 (7), 1907011. [刘冬梅, 陈秀梅, 袁泽, 问敏, 殷丽莎, 谢小吉, 黄岭. 物理化学学报, 2020, 36 (7), 1907011.] doi: 10.3866/PKU.WHXB201907011

(3) Tang, X.; Zhang, S.; Yu, J.; Lü, C.; Chi, Y.; Sun, J.; Song Y.; Yuan, D.; Ma, Z.; Zhang, L. Acta Phys. -Chim. Sin. 2020, 36 (7), 1906070. [唐小龙, 张盛辉, 于婧, 吕春晓, 迟雨晴, 孙君伟, 宋誉, 袁丁, 马兆立, 张立学. 物理化学学报, 2020,36 (7), 1906070.] doi: 10.3866/PKU.WHXB201906070

(4) Fang, B.; Feng, L. Acta Phys. -Chim. Sin. 2020, 36 (7), 1905023. [方波, 冯立纲. 物理化学学报, 2020, 36 (7), 1905023.] doi: 10.3866/PKU.WHXB201905023

(5) An, H.; Jiang, L.; Li, F.; Wu, P.; Zhu, X.; Wei, S.; Zhou, Y. Acta Phys. -Chim. Sin. 2020, 36 (7), 1905034. [安惠芳, 姜莉, 李峰, 吴平, 朱晓舒, 魏少华, 周益明. 物理化学学报, 2020,36 (7), 1905034.] doi: 10.3866/PKU.WHXB201905034

(6) Tong, Y.; Dai, M.; Xing, L.; Liu H.; Sun, W.; Wu, X. Acta Phys. -Chim. Sin. 2020, 36 (7), 1903046. [倐永丽, 戴美珍, 邢否, 刘恒岐, 孙婉婷, 武祥. 物理化学学报, 2020,36 (7), 1903046.] doi: 10.3866/PKU.WHXB201903046

(7) Wang, J.; Wu, N.; Liu, T.; Cao, S.; Yu, J. Acta Phys. -Chim. Sin. 2020, 36 (7), 1907072. [王玖, 吴南石, 刘涛, 曹少文, 余家国. 物理化学 学报, 2020, 36 (7), 1907072.] doi: 10.3866/PKU.WHXB201907072

(8) Huang, J.; Du, J.; Du, H.; Xu, G.; Yuan, Y. Acta Phys. -Chim. Sin. 2020, 36 (7), 1905056. [黄娟娟, 杜建梅, 杜海威, 徐更生, 袁玉鹏. 物理化学学报, 2020, 36 (7), 1905056.] doi: 10.3866/PKU.WHXB201905056

(9) Wang, L.; Zhu, C.; Yin, L.; Huang, W. Acta Phys. -Chim. Sin. 2020, 36 (7), 1907001. [王梁, 朱澄路, 殷丽莎, 黄维. 物理化学学报, 2020, 36 (7), 1907001.] doi: 10.3866/PKU.WHXB201907001

(10) Yu, G.; Hu, F.; Chen, W.; Han, Z.; Liu, C.; Dai, Y. Acta Phys. -Chim. $\operatorname{Sin} .2020,36$ (7), 1911016. [郁桂云, 胡丰献, 程伟伟, 韩字童, 刘超, 戴勇. 物理化学学报, 2020,36 (7), 1911016.] doi: 10.3866/PKU.WHXBWHXB201911016 